\title{
Serum levels of interleukins and S100A8/A9 correlate with clinical severity in patients with dermatomyositis-associated interstitial lung disease
}

Yueyan Lou' ${ }^{1 \dagger}$, Yu Zheng ${ }^{1 \dagger}$, Bijun Fan', Liyan Zhang ${ }^{1}$, Feng Zhu' , Xiaodong Wang ${ }^{2}$, Zhiwei Chen², Xiaoming $\operatorname{Tan}^{1 *}$ and Qing Wei ${ }^{3^{*}}$

\begin{abstract}
Background: Dermatomyositis (DM) is a systemic autoimmune inflammatory disorder that affects primarily skin, muscle and lung, frequently associated with interstitial lung disease (ILD). The objective of this study is to investigate the association between serum cytokines and clinical severity in patients with DM-ILD.

Methods: Serum samples of 30 healthy controls, 14 DM patients without ILD and 40 DM patients with ILD were collected. Serum S100A8/A9 levels were analyzed by enzyme-linked immunosorbent assay (ELISA) and levels of interleukins were measured by cytometric beads array (CBA). Then we performed multivariate logistic regression analysis to determine factors independently associated with ILD development.

Results: Serum IL-4, IL-6 and S100A8/A9 levels were significantly higher in DM patients with ILD than those in healthy controls ( $p=0.0013,0.0017$ and $<0.0001$, respectively). Serum IL-10 level of patients was dramatically lower than that in controls $(p=0.0001)$. In DM patients, the levels were significantly higher in patients with A/SIP than in those with CIP ( $p=0.0046,0.0339$ and 0.0133$)$ or without ILD ( $p=0.0165,0.0370$ and $<0.0001)$. IL-4 $(r=0.1171, p=$ $0.0040)$, IL-6 $(r=0.1174, p=0.0040)$ and IL-10 $(r=-0.1829, p=0.0003)$ were significantly correlated with S100A8/A9 in DM-ILD patients. S100A8/A9 was significantly correlated with high-resolution computed tomography (HRCT) $(r=$ $0.1642, p=0.0157$ ) and lung function (DLCO\%: $r=-0.2066, p=0.0061$, FVC\%: $r=-0.2156, p=0.0050$ ). Moreover, logistic regression analysis revealed that S100A8/A9 levels were independently associated with ILD development in DM patients $(p=0.004)$.
\end{abstract}

Conclusions: Serum level of S100A8/A9 may be a valuable predictor for assessing the clinical severity of DM-ILD patients. Serum IL-4, IL-6 and IL-10 levels were highly correlated with S100A8/A9, so these cytokines may play a synergistic effect on the progression of DM-ILD.

Keywords: Dermatomyositis, Interstitial lung disease, S100A8/A9, Interleukin

\footnotetext{
*Correspondence: tanxiaomingfsh@163.com; weiqingjn@126.com

${ }^{\dagger}$ Yueyan Lou and Yu Zheng contributed equally to this work.

'Department of Pulmonology, Renji Hospital South Campus, Shanghai

Jiaotong University School of Medicine, Shanghai, People's Republic of China

${ }^{3}$ Department of Laboratory Medicine, Renji Hospital South Campus, Shanghai Jiaotong University School of Medicine, Shanghai, People's Republic of China

Full list of author information is available at the end of the article
}

(c) The Author(s). 2020 Open Access This article is licensed under a Creative Commons Attribution 4.0 International License, which permits use, sharing, adaptation, distribution and reproduction in any medium or format, as long as you give appropriate credit to the original author(s) and the source, provide a link to the Creative Commons licence, and indicate if changes were made. The images or other third party material in this article are included in the article's Creative Commons licence, unless indicated otherwise in a credit line to the material. If material is not included in the article's Creative Commons licence and your intended use is not permitted by statutory regulation or exceeds the permitted use, you will need to obtain permission directly from the copyright holder. To view a copy of this licence, visit http://creativecommons.org/licenses/by/4.0/ The Creative Commons Public Domain Dedication waiver (http://creativecommons.org/publicdomain/zero/1.0/) applies to the data made available in this article, unless otherwise stated in a credit line to the data. 


\section{Background}

Dermatomyositis (DM) is a kind of idiopathic inflammatory myopathy (IIM), which mainly involves the inflammation of skeletal muscle and skin, and can cause muscle weakness and rash [1]. Interstitial lung disease (ILD) is considered a common systemic complication of DM [2]. DM associated with ILD is one of the major prognostic determinants, causing increased morbidity and mortality [3-5]. ILD is one of the life-threatening complications of clinically amyopathic dermatomyositis (CADM). ILD can be divided into acute/subacute interstitial pneumonia (A/SIP) and chronic interstitial pneumonia (CIP). CADM patients with A/SIP showed a rapid progressive pattern and usually resistant to intensive therapy. Therefore, early diagnosis of ILD, especially A/ SIP as a complication of CADM is critical.

Autoimmune abnormalities are commonly found in inflammatory disorders and are considered to participate in the pathogenesis of DM [6]. The inflammatory infiltrates are mainly composed of $\mathrm{T}$ lymphocytes and macrophages in muscle tissues of patients with DM. Numerous studies to date have indicated that $\mathrm{CD} 4^{+} \mathrm{T}$ helper (Th) cells may play a critical role in the pathogenesis of inflammatory myopathies [7-9]. $\mathrm{CD}^{+} \mathrm{T}$ lymphocytes are classified into type 1 (Th1) and type 2 (Th2) according to the cytokines produced. Interferon (IFN)- $\gamma$ in Th1 and interleukin (IL)- 4 in Th2 have been both measured in muscle biopsy specimens of patients with DM, suggesting the involvement of different Th subtypes in the disease [10].

Pro-inflammatory cytokines such as IL- 6 and TNF- $\alpha$ were amplified by endogenous factors such as S100 protein) $[11,12]$. These proteins are thought to be one of the "damage related molecular models" by recruiting inflammatory cells to induce inflammation [13]. S100A8 and S100A9 belong to S100 family. They are calcium binding proteins and form heterodimers. They play an important role in leukocyte trafficking and contribute to inflammatory responses [14]. S100A8/A9 is produced by infiltration of neutrophils and monocytes, rather than static macrophages or lymphocytes in the inflammatory state $[15,16]$. S100A8/A9 is an endogenous ligand of Toll like receptor 4 (TLR-4), which is related to sepsis and endotoxemia and plays an important role in innate immunity [16, 17].

The S100A8/A9 heterodimer is also referred to as calprotectin or MRP8/14. It is abundantly expressed in neutrophils and monocytes and induces proinflammatory cytokines and chemokines through TLR2/TLR4mediated signaling pathways [18]. Therefore, S100A8/A9 has been explored as a possible biomarker of disease severity in several autoimmune diseases, including juvenile dermatomyositis [19], systemic sclerosis [20] and rheumatoid arthritis [21].
The aim of this study was to investigate whether elevated serum levels of S100A8/A9 are associated with ILD development in DM patients. Furthermore, we assessed its potency as an independent biomarker to predict disease activity and to aid broad implementation into clinical practice.

\section{Methods}

\section{Participants}

The present study included 30 healthy controls, 14 DM patients without ILD and 40 DM patients with ILD. The patients were diagnosed as DM according to the classification criteria proposed by Bohan and Peter [22] [23]. Diagnosis of interstitial lung disease based on clinical symptoms and high-resolution computed tomography (HRCT), with or without lung biopsy findings. The diagnosis was based on the International Consensus Statement on Idiopathic Pulmonary Fibrosis issued by the American Thoracic Society and the European Respiratory Society [24]. Patients with ILD can be divided into two subsets: acute/subacute interstitial pneumonitis (A/ SIP) (A rapidly progressive ILD leading to respiratory function failure within 1 or 3 months) and chronic interstitial pneumonitis (CIP) (slowly progressive presentation with gradual deterioration longer than 3 months).

They were admitted to the department of Respiration and Rheumatology at our hospital from January 2017 to December 2018. Clinical and laboratory data were collected retrospectively upon admission. Exclusion criteria were immunotherapy and hormone therapy for more than 2 weeks or longer after rash, myopathy, and ILD symptoms. This study was approved by the Institutional Review Board of RenJi Hospital (2016075). All participants included consent the study orally.

\section{Measurement of cytokines}

Serum cytokine levels were detected by the $\mathrm{BD}^{\mathrm{Tm}}$ cytometric bead array (CBA) assay (BD Biosciences, San Diego, CA, U.S.A.). The Human Th1/Th2/Th17 Cytokine Kits were used to detect the serum concentrations of IL-2, IL-4, IL-6, IL-10, tumor necrosis factor- $\alpha$ (TNF$\alpha)$, IFN- $\gamma$ and IL-17A. Human MRP8/14 (Calprotectin) ELISA Kit (Biolegend, San Diego, CA, U.S.A.) was used to measure serum S100A8/A9 level.

\section{Lung high-resolution CT scoring and pulmonary function evaluation}

HRCT score was assessed by two pulmonary radiologists in a double-blind manner [25]. Each patient was tested for lung function test, percentage of predicted forced vital capacity (FVC\%) and single-breath diffusing capacity of the lung for carbon monoxide (DLCO-SB) by an experienced technician on the Jaeger platform. (CareFusion, BD Biosciences). 


\section{Statistical analysis}

Multiple unpaired t-tests were employed for comparison of serum cytokine levels between patients and healthy controls. Relationships between cytokines were investigated using Spearman's correlation coefficient test. Correlations of serum cytokines with disease severity were also using Spearman's correlation coefficient test. Univariate and multivariate logistic regression analyses were used to identify independent risk factors for ILD development. The optimal cutoff values and the area under the curve (AUC) were obtained by receiver operating characteristic (ROC) curve analysis. All the data were analyzed by SPSS 23.0 and Graphpad Prism 6.0. A Pvalue of $<0.05$ was regarded as statistically significant in all statistical analysis. The following symbols ${ }^{*} P<0.05$; * $* P<0.01 ; * P<0.001$ were used.

\section{Results}

\section{Baseline characteristic}

The present study included 30 healthy controls (11 males and 19 females; mean age $45.4 \pm 10.3$ years, range 19 to 65 ), 14 DM patients without ILD (3 males and 11 females; mean age $52.6 \pm 19.9$ years, range 17 to 80$)$ and 40 DM patients with ILD (13 males and 27 females; mean age $50.1 \pm 13.3$ years, range 22 to 78 ). In DM-ILD patients, there were 17 with A/SIP and 23 with CIP. Serological profiling of each patient, including C-reactive protein (CRP), erythrocyte sedimentation rate (ESR), Ferritin and creatine kinase (CK) was performed using the standard methods. The clinical characteristics were shown in Table 1.

\section{Comparison of serum cytokine levels between DM} patients without ILD and those with ILD

To investigate the association of levels of serum cytokines and ILD progression in DM patients, we compared their levels among DM patients with A/SIP $(n=17)$, those with CIP $(n=23)$, those without ILD $(n=14)$ and healthy controls $(n=30)$. In CBA studies, serum IL-4 $(9.743 \pm 0.7713$ versus $6.594 \pm 0.2539, P=0.0013)$ and IL-6 $\quad(44.10 \pm 8.109$ versus $11.57 \pm 1.937, \quad P=0.0017)$ levels were significantly higher in patients with DM-ILD than healthy controls. Serum IL-10 level in patients was significantly lower than controls $(1.881 \pm 0.09292$ versus $2.809 \pm 0.2445, P=0.0001)$. In ELISA analysis, DM-ILD patients had significantly higher S100A8/A9 levels than controls $(103.1 \pm 6.692$ versus $40.92 \pm 4.430, P<0.0001)$. No significant differences in the levels of IL-2, TNF- $\alpha$, IFN- $\gamma$ and IL-17A were detected between DM-ILD patients and controls (Table 2). Moreover, In DM patients, the levels of IL-4, IL-6 and S100A8/A9 were significantly higher in patients with A/SIP than in those with CIP $(p=0.0046,0.0339$ and 0.0133$)$ or without ILD $(p=$ $0.0165,0.0370$ and $<0.0001$ ) (Fig. 1).

\section{Correlation between the levels of IL-4, IL-6, IL-10 and S100A8/A9}

We next analyzed the correlation between IL-4, IL-6, IL10 and S100A8/A9 levels. As shown in Fig. 2, significant positive correlation was found between levels of IL-4, IL-6 and S100A8/A9 $\left(r_{\mathrm{s}}=0.1171, P=0.0040 ; r_{\mathrm{s}}=0.1174, P=\right.$ 0.0040). IL-10 levels were significantly negatively correlated with S100A8/A9 levels $\left(r_{\mathrm{s}}=0.1829, P=0.0003\right)$.

Table 1 Clinical characteristic of the DM-ILD patients and healthy controls

\begin{tabular}{llll}
\hline Variables & Healthy Controls $(n=30)$ & DM without ILD $(n=14)$ & DM-ILD $(n=40)$ \\
\hline Age at onset (years), mean \pm SD & $45.4 \pm 10.3$ & $52.6 \pm 19.9$ & $50.1 \pm 13.3$ \\
Gender (female/male) & $19 / 11$ & $11 / 3$ & $27 / 13$ \\
Subtype of DM (DM/CADM) & & $12 / 2$ & $18 / 22$ \\
Subtype of ILD & & & 17 \\
A/SIP in all & & & 13 \\
CIP in all & & & $144.5 \pm 37.21$ \\
Pulmonary function tests & & $84.2 \pm 14.6$ & $67.2 \pm 19.6$ \\
HRCT score & & $52.0 \pm 26.3$ & $37.4 \pm 18.5$ \\
\%FVC (\%) & & & $13.5 \pm 15.5$ \\
\%DLCO (\%) & & & $24.9 \pm 21.5$ \\
Laboratory findings & & $5.9 \pm 5.4$ & $758.7 \pm 1036.7$ \\
CRP (mg/dL) & $7.7 \pm 6.0$ & $20.6 \pm 14.4$ & $152.3 \pm 204.7$ \\
ESR (mm/h) & $172.6 \pm 147.5$ & $238.0 \pm 244.7$ & $100.0 \pm 49.3$ \\
Ferritin (ng/mL) & $75.3 \pm 29.2$ & & \\
CK (U/l) & & & \\
\hline
\end{tabular}

${ }^{a} A / S I P$ Acute/subacute interstitial pneumonia, CADM clinically amyopathic dermatomyositis, CIP Chronic interstitial pneumonia, CK creatine kinase, CRP C-reactive protein, DLCO\% \% diffusing capacity of the lungs for carbon monoxide, DM dermatomyositis, ESR erythrocyte sedimentation rate, FVC\% \% forced vital capacity, $H R C T$ high-resolution computed tomography, ILD interstitial lung disease 
Table 2 Cytokine levels of the DM-ILD patients and healthy controls

\begin{tabular}{llll}
\hline & DM-ILD $(n=40)$ Mean \pm SD, $\mathrm{pg} / \mathrm{mL}$ & Controls $(n=30)$ Mean \pm SD, pg/mL & $P$ value \\
\hline IL-2 & $4.569 \pm 0.0424$ & $4.635 \pm 0.0551$ & 0.3413 \\
IL-4 & $9.743 \pm 0.7713$ & $6.594 \pm 0.2539$ & $0.0013^{* *}$ \\
IL-6 & $44.10 \pm 8.1091$ & $11.57 \pm 1.9372$ & $0.0017^{* *}$ \\
IL-10 & $1.881 \pm 0.0929$ & $2.809 \pm 0.2445$ & $0.0001^{* * *}$ \\
TNF-a & $2.366 \pm 0.1400$ & $2.525 \pm 0.1301$ & 0.4280 \\
IFN-Y & $3.505 \pm 0.1612$ & $3.604 \pm 0.1496$ & 0.6727 \\
IL-17A & $10.525 \pm 0.1483$ & $9.367 \pm 0.0722$ & 0.5725 \\
S100A8/A9 & $103.1 \pm 6.6925$ & $40.92 \pm 4.4302$ & $<0.0001^{* * *}$ \\
\hline
\end{tabular}

**P $<0.01,{ }^{* * * P}<0.001$

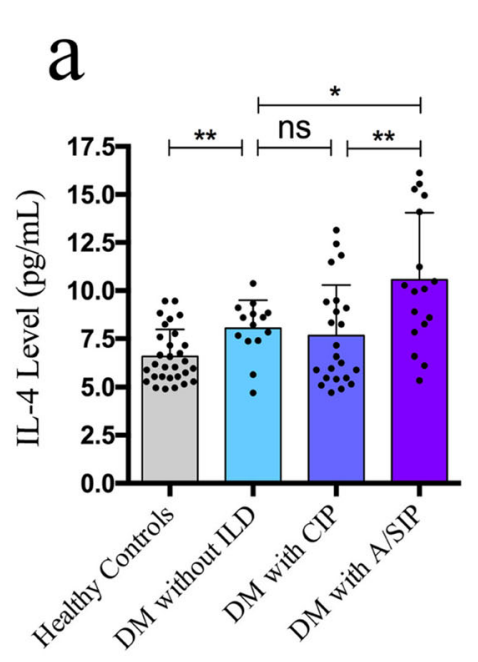

b

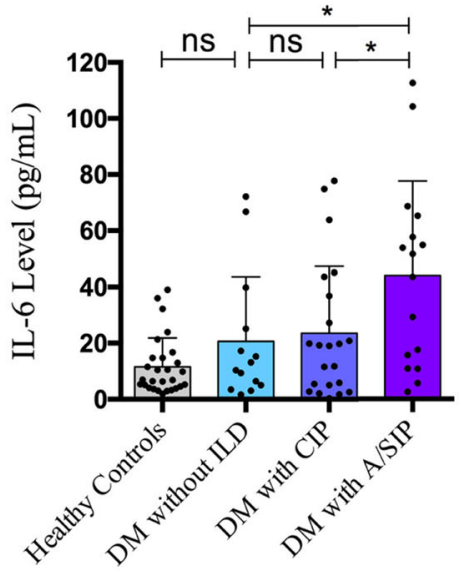

C ns
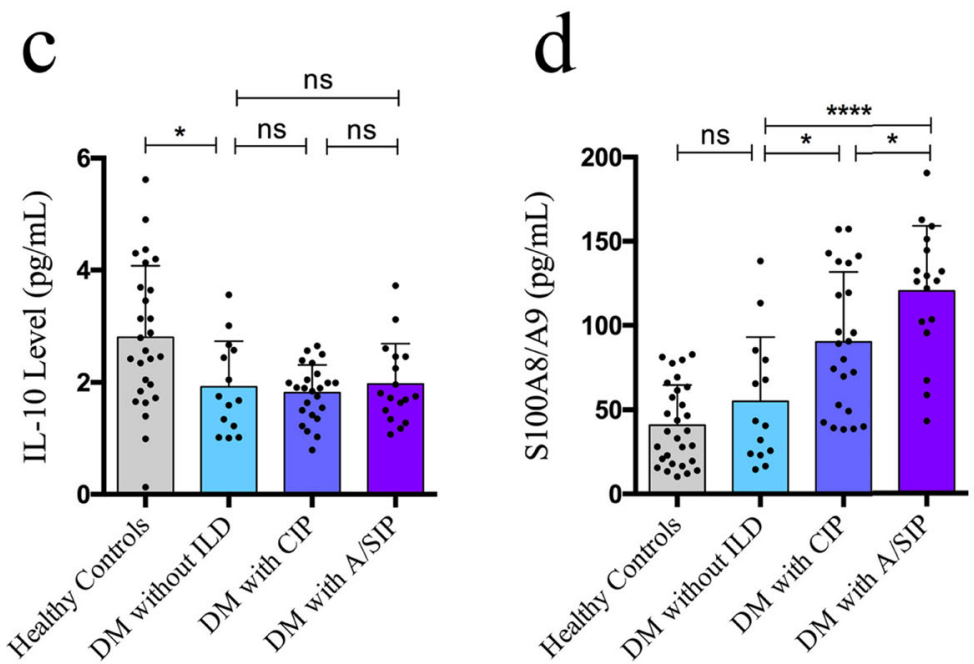

Fig. 1 Comparison of serum cytokine levels among DM patients without ILD, those with CIP and those with A/SIP. Serum S100A8/A9 levels a were determined by ELISA, and IL-4 b, IL-6 $\mathbf{c}$ and IL-10 $\mathbf{d}$ levels were analyzed by cytometric bead array (CBA). Statistical analysis was performed using the multiple unpaired t test. ${ }^{* *} P<0.01,{ }^{* *} P<0.001$ 

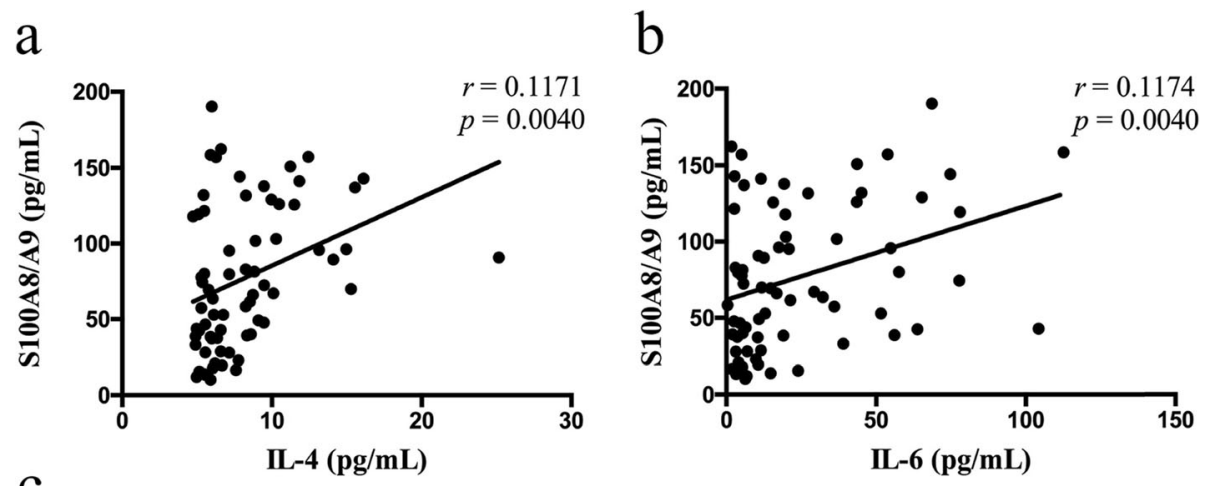

C

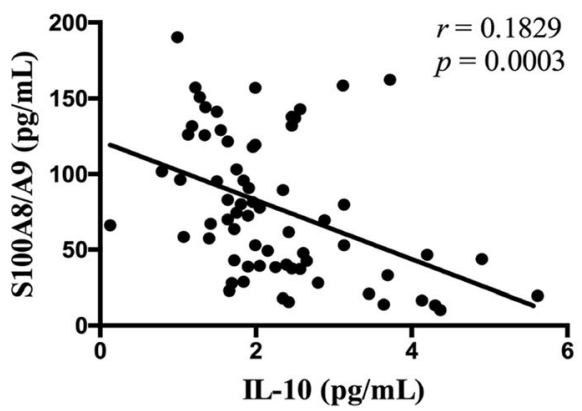

Fig. 2 Correlation between IL-4, IL-6, IL-10 and S100A8/A9 levels. The correlation analysis was performed to analyze the concentrations between S100A8/A9 and IL-4 a, IL-6 b and IL-10 $\mathbf{c}$ in patients with DM-ILD and controls

\section{Correlation between S100A8/A9 levels and disease activity in DM-ILD patients}

To assess the potency of S100A8/A9 as a biomarker to predict the disease activity of DM-ILD, we analyzed the relationship between S100A8/A9 levels and their HRCT score or lung functions in DM-ILD patients. As shown in Fig. 3, the level of S100A8/A9 was significantly positively correlated with HRCT score $\left(r_{s}=0.1642, P=0.0157\right)$ and negatively correlated with DLCO\% $\left(r_{s}=0.2066, P=0.0061\right)$ and $\mathrm{FVC} \%$ $\left(\mathrm{r}_{\mathrm{s}}=0.2156, P=0.0050\right)$.

\section{The risk factor for ILD development in DM patients}

The results of univariate and multivariate logistic regression analyses of the risk factors of ILD development in DM patients were shown in Table 3. Univariate logistic regression revealed that ILD development was significantly associated with serum S100A8/A9 levels (OR, 9.600; 95\% CI, 1.881-48.999; $p=0.007)$. Serum IL-4, IL6 levels and DLCO\% variables were not significantly associated with ILD development but were included in the multivariable base model (all $p<0.2$ ). Multivariate logistic regression demonstrated that only serum S100A8/A9 levels was a significant independent risk factor associated with ILD development (OR, 15.352; 95\% CI, 2.411$17.750 ; p=0.004)$.
Correlation between serum S100A8/A9 levels and clinical prognosis

We evaluated the association among serum S100A8/A9 levels, pulmonary function variables and prognosis of DM patients with ILD ROC curve analysis. As shown in Fig. 4, the area under the curve (AUC) value of S100A8/ A9 was 0.81 and that of S100A8/A9-HRCT- DLCO\%FVC\% 0.88 (95\% CI: 0.636-0.991, $P=0.081 ; 95 \%$ CI: $0.672-1.000, P=0.106)$. Although there was no significant difference between the single and combined features, it suggested that combined features would be more predictive of the clinical outcomes than S100A8/ A9 alone.

\section{Discussion}

DM-ILD has high morbidity and mortality. Inflammation plays a key role in the pathogenesis of DM-ILD. S100A8/A9 is mainly released by neutrophils and monocytes, and stable dimers or homodimers can be formed in vitro and in vivo. S100A8/A9 have already been verified to play an important role in the progress of inflammation. Serum S100A8/A9 levels in patients with systemic lupus erythematosus (SLE) are elevated, which may be closely related to disease activity [26, 27].. Elevated S100A9 level in sputum is a potential biomarker of neutrophilic inflammation in severe asthma [28]. Andreasson $\mathrm{K}$ et al. found that fecal S100A8/A9 level in 

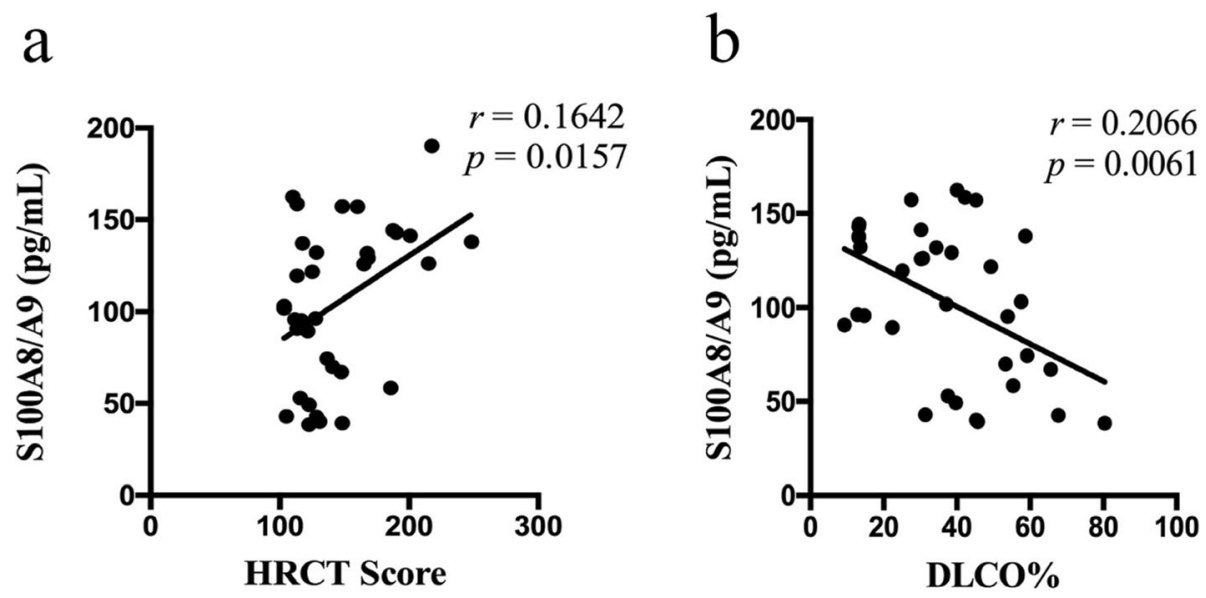

C

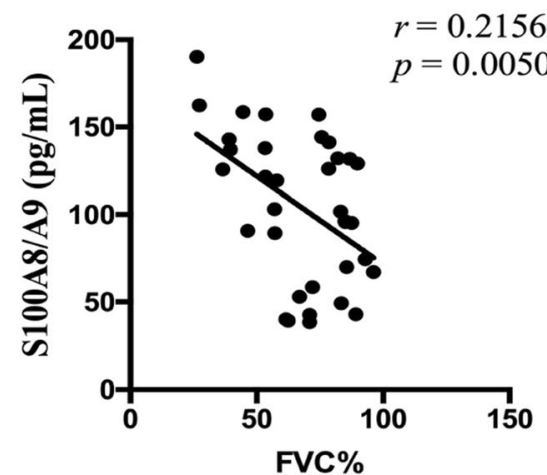

Fig. 3 Correlation between S100A8/A9 levels and disease activity in DM-ILD patients. The correlation analysis was performed to analyze the relationship between S100A8/A9 levels and HRCT score $\mathbf{a}$ and lung functions, including DLCO\% $\mathbf{b}$ and FVC\% c

Table 3 Logistic regression analysis of risk factors associated with ILD development in DM patients

\begin{tabular}{llll}
\hline Parameter & Odds ratio & $95 \%$ confidence interval & $\boldsymbol{P}$ value \\
\hline Univariate analysis & & & \\
IL-4 & 1.385 & $0.093-2.593$ & 0.188 \\
IL-6 & 2.597 & $0.628-10.743$ & 0.188 \\
IL-10 & 1.270 & $0.374-4.314$ & 0.702 \\
S100A8/A9 & 9.600 & $1.881-48.999$ & 0.007 \\
FVC\% & 0.475 & $0.125-1.808$ & 0.275 \\
DLCO\% & 0.389 & $0.107-1.411$ & 0.151 \\
C-reactive Protein & 1.833 & $0.434-7.736$ & 0.409 \\
Multivariate Analysis & & & \\
IL-4 & 1.199 & $0.032-2.233$ & 0.083 \\
IL-6 & 3.448 & $0.624-19.058$ & 0.156 \\
S100A8/A9 & 15.352 & $2.411-17.750$ & $\mathbf{0 . 0 0 4}$ \\
DLCO\% & 0.352 & $0.067-1.847$ & 0.217 \\
\hline
\end{tabular}

${ }^{\mathrm{a}} \mathrm{Cl}$ confidence interval, $\mathrm{OR}$ odds ratio patients with systemic sclerosis may be a biomarker of gastrointestinal diseases [29]. In Idiopathic pulmonary fibrosis (IPF), elevated level of S100A9 was observed in bronchoalveolar lavage fluid (BALF) [30, 31]. Hara A et al. reported that S100A9 level in BALF may be a biomarker of IPF fibrosis [32]. Therefore, based on the above researches, we hypothesized that S100A8/A9 may play a role in the development of DM-ILD.

Interleukin-4 (IL-4) is a multifunctional and multipotent cytokine, which plays an important role in proliferation, differentiation and apoptosis of various cell types, mainly secreted by mast cells, Th2 cells, eosinophils and basophils [33]. IL-6 and IL-10 had been confirmed to be associated with A/SIP in patients with DM/CADM [3436]. IL-6, IL-8, and TNF- $\alpha$ were previously indicated to be associated with overall disease activity in PM/DM [37]. However, the association between pulmonary disease activity and above cytokines has remained unclear.

To our knowledge, this is the first study to show that serum S100A8/A9 levels were significantly enhanced in DM patients with ILD, especially in those with A/SIP, compared with those without ILD. In DM-ILD patients, 


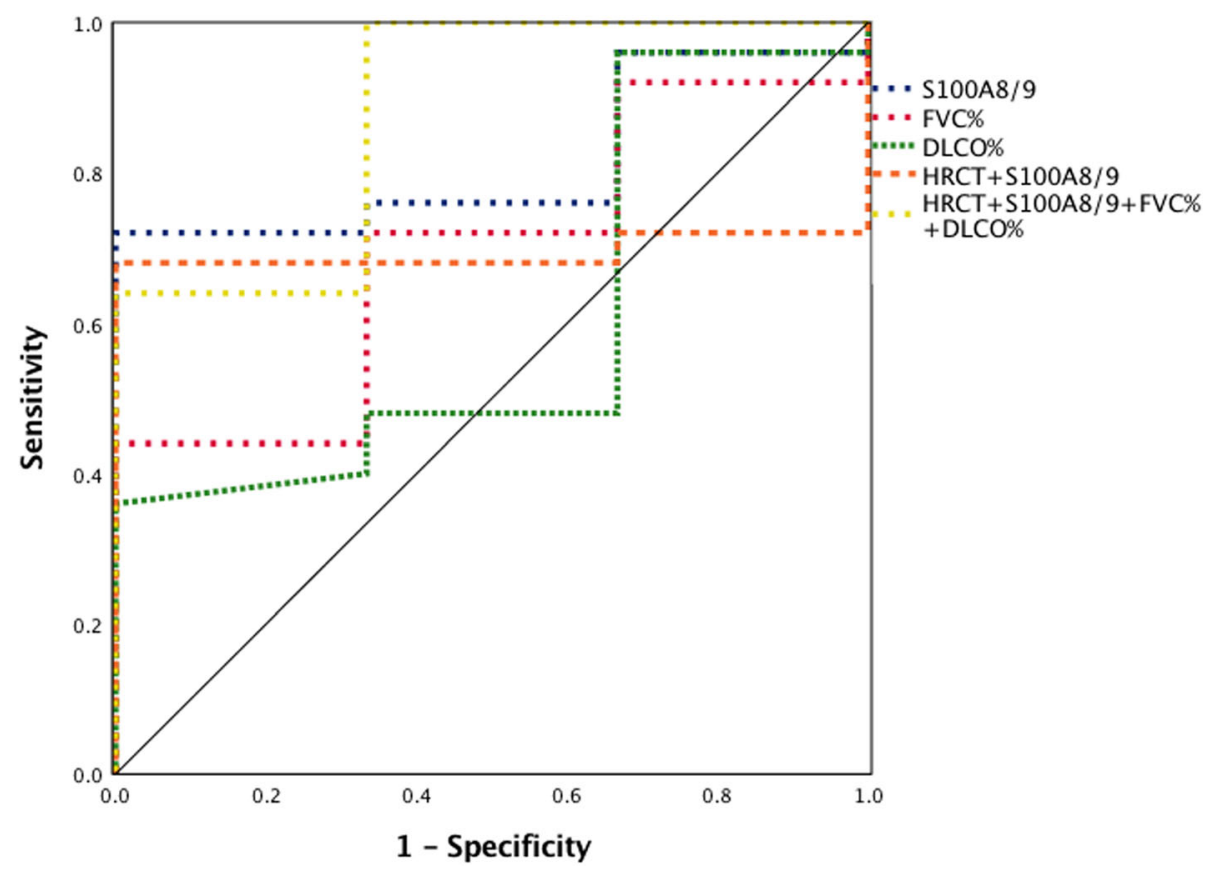

Fig. 4 Receiver operating characteristic curve analysis of serum S100A8/A9 levels, pulmonary functions and prognosis of DM patients with ILD. Receiver operating characteristic (ROC) curve analysis of 40 DM patients with ILD according to the serum S100A8/A9 levels, pulmonary functions and combined features to predict the prognosis. The combined feature has better prognostic performance in DM patients with ILD

these concentrations were associated with the disease activity and prognosis. Moreover, serum levels of IL-4, IL6 were significantly higher in DM-ILD patients than those in healthy controls $(p=0.0013,0.0017)$. Serum IL$4 \quad(r=0.1171, p=0.0040)$, IL-6 $(r=0.1174, p=0.0040)$ were significantly correlated with S100A8/A9 in patients with DM-ILD. Our findings were consistent with the previous studies that S100A8/A9, IL-4, IL-6 levels were increased in inflammatory diseases, and they may play a key role in the DM-ILD.

Different from previous studies, we found that serum IL-10 levels of DM-ILD patients were dramatically lower than controls $(p=0.0001)$. It has been indicated that IL10 family is comprised of nine members which are powerful immune mediators with versatile functions, including reducing tissue damage caused by excess and uncontrolled inflammatory effector responses [38]. Several recent studies showed that the transcription factor c-Maf up-regulated IL-10 production in vivo in CD4+ T cells from Th1, Th2, and Th17 cells in experimental disease models and Bhlhe40 repressed IL-10 production in $\mathrm{T}$ cells during immune responses [39, 40]. Thus, we hypothesize that the development of DM-ILD may be associated with the absence of c-Maf or the overexpression of Bhlhe40 in CD4+ T cells. But it remains to be confirmed in the further research.

ILD is common among patients with DM, and is often associated with a worse prognosis [41]. HRCT scoring system can be used as a parameter to evaluate the damage of ILD structure, and predict the severity and prognosis of DM-ILD [42]. Furthermore, in patients with IPF, forced vital capacity (FVC) and carbon monoxide diffusion (DLCO) are considered to be the most sensitive parameters for evaluating the course of the disease [43].. Hence, here we used FVC\% and DLCO\% as pulmonary function impairment evaluation indicators, combined with HRCT score system, to predict the severity and prognosis of DM-ILD.

We found that serum levels of S100A8/A9 were significantly correlated with ILD structural damage (HRCT score) $(r=0.1642, p=0.0157)$ and pulmonary function impairment (DLCO\%: $r=-0.2066, \quad p=$ 0.0061 , FVC\%: $r=-0.2156, p=0.0050$ ). The correlation between serum S100A8/A9 levels and HRCT and PFT impairment suggested that high serum levels of S100A8/A9 directly reflected ILD severity in patients with DM-ILD.

There are several limitations to this study. Firstly, this study was retrospectively conducted. Secondly, analysis of DM-ILD patients with anti-MDA5 antibodies was not performed. Thirdly, some patients were being treated with prednisolone at the time of serum collection. These medications maybe influenced the measurement of cytokine levels. Further studies are required to investigate the precise mechanisms by which S100A8/A9 contributes to DM-ILD. 


\section{Conclusions}

In conclusion, this study demonstrated that serum S100A8/A9 levels were elevated in DM patients with ILD, in particular those with A/SIP. These levels were highly correlated with IL-4, IL-6 and IL-10 levels and were significantly correlated with HRCT score, FVC\% and DLCO\%. These cytokines may contribute to the pathogenesis of DM-ILD. Our results suggest that S100A8/A9 may be a useful biomarker for assessing ILD activity and predicting prognosis in DM patients with ILD.

\section{Abbreviations}

A/SIP: Acute/subacute interstitial pneumonia; BALF: Bronchoalveolar lavage fluid; CADM: Clinically amyopathic dermatomyositis; CBA: Cytometric beads array; CIP: Chronic interstitial pneumonia; CK: Creatine kinase; CRP: C-reactive protein; DLCO\%: \% Diffusing capacity of the lungs for carbon monoxide; DM: Dermatomyositis; ELISA: Enzyme-linked immunosorbent assay; ESR: Erythrocyte sedimentation rate; FVC\%: \% Forced vital capacity; GGO: Ground glass opacities; HRCT: High-resolution computed tomography; IFN: Interferon; IIM: Idiopathic inflammatory myopathy; IL: Interleukin; ILD: Interstitial lung disease; IPF: Idiopathic pulmonary fibrosis; Th: T-helper; TLR: Toll-like receptor; TNF- a: Tumor necrosis factor- a; SLE: Systemic lupus erythematosus

\section{Acknowledgements}

Not Applicable.

\section{Authors' contributions}

All authors were involved in drafting the article or revising it critically for important intellectual content, and all authors approved the final version to be submitted for publication. XT and QW had full access to all of the data in the study and take responsibility for the integrity of the data and the accuracy of the data analysis. All authors read and approved the final versions of the manuscript and supplemental files. Study conception and design: YL, YZ, XW, XT, QW. Acquisition of the data: YL, LZ, FZ, ZC. Analysis and interpretation of the data: $Y L, B F, Y Z, Q W$.

\section{Funding}

This work was supported by grants from Shanghai Health and Family Planning Commission clinical research project (No 20184Y0189) and a study on the diagnosis and treatment strategy of interstitial lung disease associated with connective tissue in diagnostic bronchoscopy (No PYI-17009). The funding body had no role in the study design, the collection, analysis or interpretation of the data.

\section{Availability of data and materials}

The dataset used and/or analyzed during the current study are available from the corresponding author on reasonable request.

\section{Ethics approval and consent to participate}

The study was approved by the Institutional Review Board of RenJi Hospital (2016075). Since all data were anonymously used and this study does not contain protected health information, the ethics committee approved a waiver of the requirement for informed consent. Individual patients consent was not obtained since all data used in this study were acquired retrospectively from the laboratory information system without any additional sampling or laboratory analysis. Patients could choose to opt out at any time and have their data removed from the registry.

\section{Consent for publication}

Not Applicable.

\section{Competing interests}

The authors have declared no conflicts of interest.

\section{Author details}

'Department of Pulmonology, Renji Hospital South Campus, Shanghai Jiaotong University School of Medicine, Shanghai, People's Republic of China. ${ }^{2}$ Department of Rheumatology, Renji Hospital South Campus, Shanghai Jiaotong University School of Medicine, Shanghai, People's Republic of China. ${ }^{3}$ Department of Laboratory Medicine, Renji Hospital South Campus, Shanghai Jiaotong University School of Medicine, Shanghai, People's Republic of China.

Received: 3 September 2019 Accepted: 2 July 2020

Published online: 17 July 2020

\section{References}

1. Lega JC, et al. Idiopathic inflammatory myopathies and the lung. Eur Respir Rev. 2015;24(136):216-38.

2. Hallowell RW, Ascherman DP, Danoff SK. Pulmonary manifestations of polymyositis/dermatomyositis. Semin Respir Crit Care Med. 2014;35(2):23948.

3. Hanaoka M, et al. KL-6 is a long-term disease-activity biomarker for interstitial lung disease associated with polymyositis/dermatomyositis, but is not a short-term disease-activity biomarker. Mod Rheumatol. 2018:1-20.

4. Li L, et al. Myositis-specific autoantibodies in dermatomyositis/polymyositis with interstitial lung disease. J Neurol Sci. 2019;397:123-8.

5. Morisset J, et al. Management of Myositis-Related Interstitial Lung Disease. Chest. 2016;150(5):1118-28.

6. Grundtman C, Malmstrom V, Lundberg IE. Immune mechanisms in the pathogenesis of idiopathic inflammatory myopathies. Arthritis Res Ther. 2007;9(2):208

7. Moran EM, Mastaglia FL. The role of interleukin-17 in immune-mediated inflammatory myopathies and possible therapeutic implications. Neuromuscul Disord. 2014;24(11):943-52.

8. Shimojima Y, et al. Phenotypes of peripheral blood lymphocytes and cytokine expression in Polymyositis and Dermatomyositis before treatment and after clinical remission. Clin Med Insights Arthritis Musculoskelet Disord. 2012;5:77-87.

9. Giris M, et al. Elevated IL-4 and IFN-gamma levels in muscle tissue of patients with Dermatomyositis. Vivo. 2017;31(4):657-60.

10. Fujiyama T, et al. Preferential infiltration of interleukin-4-producing CXCR4+ T cells in the lesional muscle but not skin of patients with dermatomyositis. Clin Exp Immunol. 2014;177(1):110-20.

11. Rasheed Z, Akhtar N, Haqqi TM. Advanced glycation end products induce the expression of interleukin- 6 and interleukin- 8 by receptor for advanced glycation end product-mediated activation of mitogen-activated protein kinases and nuclear factor-kappaB in human osteoarthritis chondrocytes. Rheumatology (Oxford). 2011;50(5):838-51.

12. Guloksuz SA, et al. Elevated plasma concentrations of S100 calcium-binding protein $B$ and tumor necrosis factor alpha in children with autism spectrum disorders. Braz J Psychiatry. 2017;39(3):195-200.

13. Roth J, et al. Phagocyte-specific $\mathbf{S 1 0 0}$ proteins: a novel group of proinflammatory molecules. Trends Immunol. 2003;24(4):155-8.

14. Edgeworth J, et al. Identification of p8,14 as a highly abundant heterodimeric calcium binding protein complex of myeloid cells. J Biol Chem. 1991;266(12):7706-13.

15. Raquil MA, et al. Blockade of antimicrobial proteins S100A8 and S100A9 inhibits phagocyte migration to the alveoli in streptococcal pneumonia. J Immunol. 2008;180(5):3366-74.

16. Vogl T, et al. Mrp8 and Mrp14 are endogenous activators of toll-like receptor 4, promoting lethal, endotoxin-induced shock. Nat Med. 2007;13(9): 1042-9.

17. van Zoelen MA, et al. Expression and role of myeloid-related protein-14 in clinical and experimental sepsis. Am J Respir Crit Care Med. 2009;180(11): 1098-106.

18. Liang $\mathrm{X}$, et al. Platelet-neutrophil interaction aggravates vascular in $\mathrm{fl}$ ammation and promotes the progression of atherosclerosis by activating the TLR4/NF-kappaB pathway. J Cell Biochem. 2019;120(4):5612-9.

19. Nistala K, et al. Myeloid related protein induces muscle derived inflammatory mediators in juvenile dermatomyositis. Arthritis Res Ther. 2013;15(5):R131.

20. Hesselstrand $\mathrm{R}$, et al. Biomarkers from bronchoalveolar lavage fluid in systemic sclerosis patients with interstitial lung disease relate to severity of lung fibrosis. Respir Med. 2013;107(7):1079-86. 
21. Yunchun $L$, et al. Clinical significance of myeloid-related protein $8 / 14$ as a predictor for biological treatment and disease activity in rheumatoid arthritis. Ann Clin Lab Sci. 2018;48(1):63-8.

22. Bohan A, Peter JB. Polymyositis and dermatomyositis (second of two parts). N Engl J Med. 1975;292(8):403-7.

23. Bohan A, Peter JB. Polymyositis and dermatomyositis (first of two parts). N Engl J Med. 1975;292(7):344-7.

24. American Thoracic Society. Idiopathic pulmonary fibrosis: diagnosis and treatment. International consensus statement. American Thoracic Society (ATS), and the European Respiratory Society (ERS). Am J Respir Crit Care Med. 2000;161(2 Pt 1):646-64.

25. Ikezoe J, et al. High-resolution CT findings of lung disease in patients with polymyositis and dermatomyositis. J Thorac Imaging. 1996;11(4):250-9.

26. Wang $\mathrm{Y}$, et al. Platelet-derived S100 family member myeloid-related protein14 regulates thrombosis. J Clin Invest. 2014;124(5):2160-71.

27. Tyden $\mathrm{H}$, et al. Pro-inflammatory S100 proteins are associated with glomerulonephritis and anti-dsDNA antibodies in systemic lupus erythematosus. Lupus. 2017;26(2):139-49.

28. Lee TH, Song HJ, Park CS. Role of inflammasome activation in development and exacerbation of asthma. Asia Pac Allergy. 2014;4(4):187-96.

29. Andreasson $\mathrm{K}$, et al. Faecal calprotectin: a biomarker of gastrointestinal disease in systemic sclerosis. J Intern Med. 2011:270(1):50-7.

30. Korthagen NM, et al. MRP14 is elevated in the bronchoalveolar lavage fluid of fibrosing interstitial lung diseases. Clin Exp Immunol. 2010;161(2):342-7.

31. Bargagli E, et al. Macrophage-derived biomarkers of idiopathic pulmonary fibrosis. Pulm Med. 2011;2011:717130.

32. Hara A, et al. S100A9 in BALF is a candidate biomarker of idiopathic pulmonary fibrosis. Respir Med. 2012;106(4):571-80.

33. Gadani SP, et al. IL-4 in the brain: a cytokine to remember. J Immunol. 2012; 189(9):4213-9.

34. Gono T, et al. Cytokine profiles in polymyositis and dermatomyositis complicated by rapidly progressive or chronic interstitial lung disease Rheumatology (Oxford). 2014;53(12):2196-203.

35. Gono T, et al. Interleukin-18 is a key mediator in dermatomyositis: potential contribution to development of interstitial lung disease. Rheumatology (Oxford). 2010;49(10):1878-81.

36. Kawasumi H, et al. IL-6, IL-8, and IL-10 are associated with hyperferritinemia in rapidly progressive interstitial lung disease with polymyositis/ dermatomyositis. Biomed Res Int. 2014;2014:815245.

37. Reed AM, et al. Changes in novel biomarkers of disease activity in juvenile and adult dermatomyositis are sensitive biomarkers of disease course. Arthritis Rheum. 2012;64(12):4078-86.

38. Ouyang $W$, et al. Regulation and functions of the IL-10 family of cytokines in inflammation and disease. Annu Rev Immunol. 2011;29:71-109.

39. Gabrysova L, et al. C-Maf controls immune responses by regulating diseasespecific gene networks and repressing IL-2 in CD4(+) T cells. Nat Immunol. 2018;19(5):497-507.

40. Lin CC, et al. Bhlhe40 controls cytokine production by T cells and is essential for pathogenicity in autoimmune neuroinflammation. Nat Commun. 2014:5:3551.

41. Marie I, et al. Short-term and long-term outcomes of interstitial lung disease in polymyositis and dermatomyositis: a series of 107 patients. Arthritis Rheum. 2011;63(11):3439-47.

42. Bonnefoy $\mathrm{O}$, et al. Serial chest $\mathrm{CT}$ findings in interstitial lung disease associated with polymyositis-dermatomyositis. Eur J Radiol. 2004:49(3):23544.

43. Ley B, Collard HR, King TE Jr. Clinical course and prediction of survival in idiopathic pulmonary fibrosis. Am J Respir Crit Care Med. 2011;183(4):43140.

\section{Publisher's Note}

Springer Nature remains neutral with regard to jurisdictional claims in published maps and institutional affiliations.

Ready to submit your research? Choose BMC and benefit from:

- fast, convenient online submission

- thorough peer review by experienced researchers in your field

- rapid publication on acceptance

- support for research data, including large and complex data types

- gold Open Access which fosters wider collaboration and increased citations

- maximum visibility for your research: over $100 \mathrm{M}$ website views per year

At $\mathrm{BMC}$, research is always in progress.

Learn more biomedcentral.com/submissions 